\title{
Article
}

\section{Fonctions multiples, contraintes multiples: les défis du nouveau paradigme de gestion des zones alluviales suisses}

\author{
Mihaela Nedelcu ${ }^{a}$, François Hainard ${ }^{a}$, Dominique Guex ${ }^{b}$, Jean-Michel Gobat ${ }^{c}$ \\ a Sociologues, Institut de sociologie, Faubourg de l'Hôpital 27, 2000 Neuchâtel, Suisse \\ b Hydrologue, EPFL, Faculté ENAC, HYDRAM, 1015 Lausanne, Suisse \\ c Écologue, Université de Neuchâtel, Laboratoire Sol et Végétation, rue Émile-Argand 11, Case postale 158, 2009 Neuchâtel, Suisse
}

\begin{abstract}
En Suisse, les approches actuelles de la gestion des écosystèmes fluviaux reposent sur deux principes d'action complémentaires : assurer la sécurité des populations et des infrastructures et conserver la biodiversité des zones alluviales. Ces approches essaient de dépasser les limites des modèles antérieurs, qui revenaient plutôt à imposer unilatéralement les impératifs d'une filière (l'évacuation des crues ou l'hydro-électricité, par exemple), ou d'une fonction, au détriment d'une gestion intégrée à l'échelle d'un territoire. Les auteurs montrent ici que le nouveau paradigme de gestion des zones alluviales, parce qu'il s'efforce de combiner les attentes de plusieurs filières et fonctions, exige des capacités accrues pour faire aboutir une gestion collective de ces espaces. En aidant à mesurer l'ampleur du défi, en pointant des questions non résolues, cet article suggère des leçons intéressantes pour tous les types d'écosystèmes.
\end{abstract}

La Rédaction

\section{Mots-clés :}

gestion

environnementale; zone alluviale ; interdisciplinarité; étude de cas ;

Suisse

\section{Keywords:} environmental management; floodplain; interdisciplinarity; case study; Switzerland

\begin{abstract}
Résumé - Les zones alluviales constituent un objet environnemental complexe, très révélateur des enjeux sécuritaires, culturels, économiques et écologiques à l'œuvre dans la gestion actuelle des cours d'eau. Cet article, construit sur les résultats d'une recherche interdisciplinaire récente, interroge, à travers trois études de cas, sur la transformation des modes de gestion des cours d'eau en Suisse. Articulant dorénavant les principes de sécurité et de conservation de la biodiversité, cette transformation prend en compte les contraintes écologiques, hydrologiques et socioculturelles qui délimitent l'action publique dans le domaine de la gestion des cours d'eau. L'émergence des nouvelles procédures de communication, de médiation et de négociation est au cœur d'un processus d'apprentissage social collectif qui devrait, à terme, produire un changement des valeurs, des représentations sociales de la nature et des risques, ainsi que des principes qui orientent l'action.
\end{abstract}

\begin{abstract}
Multiple functions, multiple constraints: the challenges facing the new management paradigm for Swiss floodplains. Floodplains are a complex environmental "object" which reveal the cultural, economic and ecological as well as safety stakes nowadays to be taken into account in river management. This paper is based on the results of a recent interdisciplinary research involving scientists in sociology, hydrology and soil ecology. Taking three case studies it examines the changes in the river management paradigm in Switzerland. This new paradigm pays growing attention to environmental protection issues and complementarily articulates safety and biodiversity conservation principles. Its application is nevertheless faced with various ecological, hydrological and socio-cultural constraints that limit public action. Floodplains represent a multidimensional ecosystem whose natural dynamic is crucial for maintaining the richness of its biodiversity. In this perspective, the new paradigm aims to reconcile ecological concerns with modern hydrological techniques and public acceptance. Complex social negotiations govern the implementation of the new revitalization measures. Public and private actors are the main pivots of these processes. New communication, collaboration and mediation procedures are enabling a process of social collective training. This process could facilitate in the future a deeper change in the values, the social representations of nature and risks, and the principles of value-based action.
\end{abstract}

Auteur correspondant:M. Nedelcu, mihaela.nedelcu@unine.ch 


\section{Introduction}

Au cours des deux derniers siècles, la dynamique et la morphologie des cours d'eau ont subi une transformation profonde. Le paradigme de gestion des plaines alluviales ${ }^{1}$, bâti au XIX ${ }^{\mathrm{e}}$ siècle sur les exigences de sécurité (à la fois hydrologique, énergétique, sanitaire et alimentaire) de la société, s'est traduit par une série de corrections et d'aménagements lourds de conséquences sur le fonctionnement des systèmes alluviaux (Schmid, 2002; Vischer, 2003). Une étude des cartes topographiques au 1/25 000 a montré que la surface des zones alluviales suisses a diminué de $90 \%$ depuis 1850 et que leurs fonctions naturelles ont été fortement perturbées (Müller-Wenk et al., 2004a; OFS, 2002). Au regard de ces transformations radicales et de l'importance écologique évidente des zones alluviales, la mise en place de nouveaux concepts de gestion devient nécessaire (Naiman et al., 2005 ; Vannote et al., 1980 ; Pautou, G., 1988; Pautou et al., 1996 ; Piégay et al., 2003). À partir des années 1970, le principe de sécurité à l'œuvre dans les politiques de gestion des cours d'eau est constamment questionné (Willi et al., 2001, Schumm, 1977). Un nouveau modèle de gestion émerge, articulant - dans un souci de complémentarité - la sécurité et la conservation de la nature. Si, en Suisse, ce principe de complémentarité semble acquis au niveau de la législation et de sa mise en œuvre par l'administration fédérale, sa traduction dans l'action publique se heurte cependant à de nombreuses difficultés, d'ordres culturel, technique, économique et bureaucratique. Cet article interroge, à partir d'une recherche interdisciplinaire ${ }^{2}$ qui a réuni trois disciplines - sociologie, hydrologie et écologie-pédologie - autour de la thématique commune de la gestion des zones alluviales, sur les conditions d'acceptation et de mise en pratique du nouveau paradigme de gestion (Encadré).

\section{Exigences objectives et limites subjectives de l'agir}

Afin de répondre au double impératif de sécurité et de conservation de la biodiversité, le changement de

\footnotetext{
1 Par convention, la plaine alluviale comprend l'ensemble du fond de vallée mis en place par les processus de sédimentation et d'érosion fluviale, quelle que soit son occupation actuelle. La zone alluviale représente la portion de la plaine alluviale toujours soumise aux influences, plus ou moins directes, du cours d'eau (milieux encore en dynamique active, forêts en voie de stabilisation, par exemple).

${ }^{2}$ Cette recherche porte le titre « Les plaines alluviales de l'arc alpin entre sécurité et biodiversité : changement des représentations, des décisions et des pratiques des intervention » (projet Flood'Alps) et a été menée dans le cadre du programme national de recherche PNR48 (Paysage et habitat dans l'arc alpin) du Fond national suisse de la recherche (http://www.pnr48.ch). Elle a donné lieu à plusieurs publications, dont notamment l'ouvrage de M. Nedelcu et F. Hainard (2006).
}

Encadré . Les terrains d'étude et l'enquête sociologique

\section{Les terrains}

Trois sites d'étude concernant quatre rivières suisses : la Sarine (canton de Fribourg, FR), le Rhin (canton des Grisons, GR), le Ticino (canton du Tessin, TI) et la Moesa (cantons du Tessin, TI, et des Grisons, GR). Il s'agit de cours d'eau moyens, ayant subi une série de corrections et d'aménagements qui les rendent assez typiques de la situation des rivières suisses.

Le choix des sites a été déterminé par plusieurs critères complémentaires : l'existence de travaux de recherche permettant un suivi écologique et hydrologique des plaines alluviales retenues, l'état d'avancement dans la mise en pratique des nouvelles directives de protection contre les crues (dont la revitalisation) et la diversité sociolinguistique des trois régions.

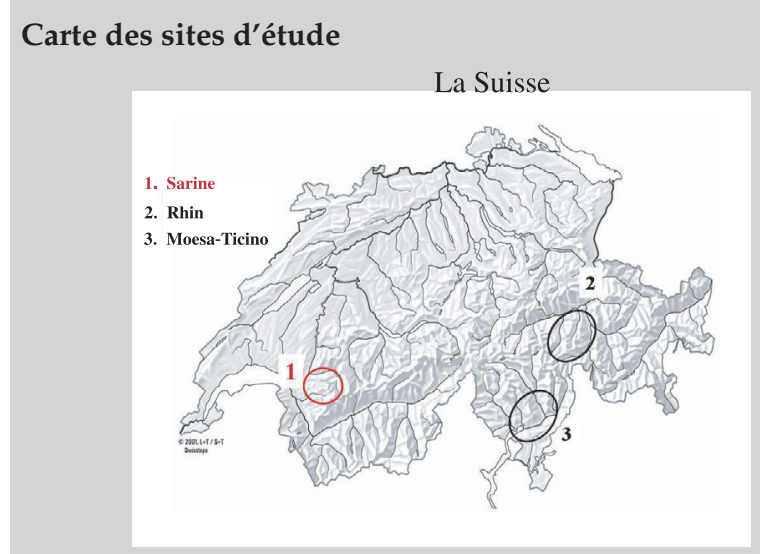

L'enquête sociologique

Les objectifs de cette recherche - repérer des valeurs, des représentations de risques, des idéologies, des légitimations et des adhésions à des mesures actuelles et surtout prospectives ont exigé une approche méthodologique de type qualitatif. Plusieurs techniques d'enquête ont été conjuguées : analyse documentaire, collecte de données par entretien semi-directif et analyse de contenu, stimuli visuels, etc.

L'enquête par entretien a été menée auprès d'une quarantaine de personnes, dont une vingtaine de décideurs et de professionnels. Plusieurs paramètres ont déterminé le choix de la population interrogée : le sexe, l'âge, la langue, la formation et la possession de biens immobiliers et/ou fonciers et leur exploitation à but professionnel dans les zones concernées. L'essentiel des entretiens a porté sur les représentations (de l'environnement, de la nature, du risque, de la science, etc.), les légitimités (des interventions, des décisions, des scientifiques et des politiques, etc.) et les scénarios (prospectifs) auxquels les résidents adhéreraient (ou refuseraient d'adhérer) selon leurs connaissances, leurs intérêts, leur conscience des problèmes et leur compréhension des enjeux.

paradigme demande des transformations importantes au niveau des comportements de chacun, de même que des modes et des techniques d'intervention. Ces transformations sont à la fois déterminées et limitées par les différentes exigences et contraintes, tant physiques - objectives (principalement écologiques et hydrologiques) que culturelles - subjectives, à l'œuvre dans la gestion du système alluvial. 


\section{Les exigences écologiques}

Les écosystèmes alluviaux sont, sous les climats européens, les plus riches en biodiversité ${ }^{3}$ (Tockner et Ward, 1999; Müller-Wenk et al., 2004b ; Ward et al., 1998). Cette richesse découle d'un paradoxe apparent : la plaine alluviale, considérée ici comme l'écosystème de niveau supérieur, est constituée d'une mosaïque hétérogène de milieux homogènes. À l'échelle de la plaine entière, cohabitent de nombreux écosystèmes variés, allant de l'eau libre stagnante ou courante aux forêts pionnières ou stabilisées, en passant par des cultures, des prairies ou des villes, ou encore des gravières ou des bosquets. Cette mosaïque reflète autant les contraintes et les perturbations hydrologiques (inondations, crues, sédimentation, érosion) que celles de l'activité humaine proprement dite. À une échelle inférieure, chacun de ces milieux évolue également pour lui-même, acquérant sa propre spécificité, avec ses conditions écologiques locales, ses espèces animales et végétales caractéristiques, ses qualités individuelles de résistance, de résilience ou de fonctionnement trophique.

Globalement, la plaine alluviale apparaît donc comme un écosystème tridimensionnel particulièrement bien organisé (Ward, 1989; Amoros et Petts, 1993), à l'origine d'une biodiversité élevée :

- la dimension longitudinale se réfère à tous les phénomènes résultant des flux unidirectionnels le long du gradient amont-aval;

- la dimension transversale considère les éléments disposés à la surface de la plaine alluviale, entre le cours principal et les forêts alluviales les plus éloignées ;

- la dimension verticale concerne la superposition des écosystèmes superficiels (pelouses, steppes, etc.) et souterrains (nappes latérales), ceux-ci faisant la liaison avec les écosystèmes aquatiques (bras morts, lits des cours d'eau, etc.).

À cette organisation tridimensionnelle structurelle, il faut encore ajouter une quatrième dimension qui agit comme moteur de la dynamique du système : l'évolution temporelle des processus physiques et écologiques (Ward, 1989). Certes, tous les écosystèmes sont organisés dans le temps; mais la plaine alluviale constitue un modèle dans lequel les processus dynamiques représentatifs de toutes les échelles de temps sont concentrés sur des surfaces souvent relativement petites, et donc en très

\footnotetext{
${ }^{3}$ On entend souvent par biodiversité la diversité d'écosystèmes (variétés d'habitats qui abritent des organismes vivant dans une région géographique particulière), la diversité d'espèces (variétés d'espèces propres à un écosystème) et la diversité génétique (la complexité d'information génétique codée dans l'ADN de la population d'une seule espèce, qui détermine la capacité de l'organisme à s'adapter aux changements d'environnement).
}

forte interaction. La plaine alluviale est ainsi organisée en échelles temporelles très fortement imbriquées, allant d'événements brefs et aléatoires, les crues, entraînant des perturbations plus ou moins importantes du système, à des modifications plus profondes et de plus longue durée, comme la perturbation du régime hydrosédimentaire à la suite du changement climatique ou des transformations anthropiques des conditions amont (Liébault et Piégay, 2001; Descroix et Gautier, 2002). D'une autre manière, on peut dire que la stabilité globale de la plaine alluviale est faite de l'addition de multiples instabilités locales (Ellenberg, 1988).

Pour le maintien de la biodiversité très élevée de la plaine alluviale, l'élément fondamental est ainsi la persistance à long terme de cette organisation quadridimensionnelle hiérarchisée, tant dans l'espace que dans le temps. Ainsi respecté, ce principe de base garantit leur place à l'ensemble des écosystèmes de rang inférieur, qu'ils soient situés en amont ou en aval dans la plaine, sis à proximité du cours d'eau ou non, aquatiques ou terrestres, jeunes et fréquemment renouvelés, ou matures et proches du climax.

D'autres contraintes, plus particulières mais tout autant indispensables, découlent de ce principe :

- une surface globale de la plaine alluviale suffisamment importante pour assurer la présence d'un nombre maximum de sous-unités ;

- un arrangement spatial des écosystèmes favorisant les échanges d'espèces et de gènes (connectivité biologique), à l'intérieur de la plaine mais aussi avec les éléments paysagers adjacents ;

- des surfaces respectives suffisamment grandes pour chacune des sous-unités écologiques, leur garantissant des aires minimales pour leur fonctionnement trophique-dynamique interne (ressources, espèces) ;

- une connectivité hydraulique la meilleure possible en amont et en aval de la plaine pour assurer la « recharge » régulière en espèces, notamment les espèces aquatiques, à partir de réservoirs génétiques externes;

- une activité humaine respectant le principe fondamental de préservation des plaines alluviales, c'est-àdire laissant dans chacune d'elles des conditions de développement potentiel à chaque sous-unité.

Bien mis en évidence dans des situations existantes, notamment dans les plaines alluviales étudiées dans notre recherche, ce principe et les contraintes subséquentes sont évidemment applicables directement aux projets de revitalisation. Dans ce cas toutefois, les aspects hydrologiques, hydrauliques, sociaux, économiques et techniques doivent évidemment être intégrés à la démarche (Bravard et al., 1986; Hary et Nachtnebel, 1989; Naiman et Bilby, 1998; Décamps et Décamps, 2002; Malavoi, 1998; Piégay, 1997). 


\section{Les exigences hydrologiques}

Si la loi fédérale sur la police des eaux de 1877, première base légale permettant le subventionnement de travaux de correction de cours d'eau, représentait à l'époque l'expression d'une nouvelle vision de la protection contre les crues (non plus locale mais à plus grande échelle), susceptible de créer les conditions de base pour une meilleure protection contre les risques d'inondation, elle est aussi à l'origine d'une transformation radicale des paysages des vallées alpines et de leurs régimes hydrologiques. Par exemple, l'incision des lits fluviaux, processus souhaité dans un premier temps, s'est généralisée et accentuée, atteignant des amplitudes de plus de 10 mètres sur le profil en long de certains cours d'eau alpins (Landon et al., 1998; Peiry et al., 1994; Surian et Rinaldi, 2003). Elle est généralement due à l'exploitation de la ressource eau et de la ressource sol. Cette exploitation s'est traduite par des travaux de recalibrage, d'endiguement et de rectification des cours d'eau, qu'ils soient de type à méandres ou en tresses, ainsi que par la construction d'ouvrages de rétention. Le transfert rapide des écoulements au travers des tronçons rectifiés et l'érosion verticale du lit des cours d'eau se sont révélés à l'originine de problèmes majeurs à partir des années 1950. Sur un plan socioéconomique, les impacts de ces modifications sont : (i) la déstabilisation des ouvrages d'art (ponts, digues), qui nécessite des travaux de reconstruction ou de protection forts coûteux; (ii) la réduction du volume de l'aquifère alluvial, qui entraîne la perte d'un stock d'eau potable de qualité ; (iii) et surtout l'accentuation des pointes de crue en aval des cours d'eau canalisés, entraînant de nouveaux débordements. Sur le plan écologique, la perturbation des processus d'ajustement morphodynamique a contribué à modifier durablement le fonctionnement des écosystèmes (Bravard et al., 1986; Pautou et al., 1992; Girel, 1991; Gallandat et al., 1993). On a ainsi pu observer la modification des peuplements végétaux riverains par suite de l'abaissement de la nappe alluviale (Girel, loc. cit.; Gallandat et al., loc. cit.), une perte de biodiversité et la banalisation des milieux.

À partir des années 1970, la prise de conscience des impacts environnementaux a commencé à modifier la conception de l'aménagement des cours d'eau, jusqu'alors essentiellement sécuritaire et structurelle. La théorie des systèmes qui s'est diffusée au sein de la communauté scientifique depuis cette époque a permis de lier processus abiotiques et biotiques et a eu une influence déterminante sur le développement de la géomorphologie fluviale, notamment (Chorley et Kennedy, 1971; Bennett et Chorley, 1978). Concept intégrateur de la diversité des processus environnementaux qui agissent à l'échelle du bassin versant, cette théorie devient un outil important d'organisation de la recherche. Le système fluvial est un modèle conceptuel du fonctionnement géomorphologique d'un bassin versant axé sur l'organisation spatiale des transferts sédimentaires en son sein (Schumm, 1977). Il met l'accent sur les processus, les modes de circulation de l'eau, les phénomènes d'interfaces et prend en compte la globalité et la spécificité d'un espace géographique en intégrant toutes les interférences, y compris les influences humaines. Ce concept fédérateur a servi de support à la recherche et permis de mieux comprendre le fonctionnement de tous les compartiments constituant le système fluvial.

De cette évolution vers une sensibilité environnementale tant de la société que des milieux scientifiques et politiques est née la loi fédérale sur l'aménagement des cours d'eau en 1991, expression d'une volonté de protection durable contre les crues. Parmi ses principes fondamentaux, notons d'une part l'idée que, dans un contexte où la sécurité absolue par des ouvrages de protection n'existe pas, il faut redonner de l'espace au cours d'eau, pour retenir une partie du volume des crues et écrêter leurs pointes en créant des zones inondables; d'autre part, l'espace fluvial est reconnu en tant qu'espace multifonctionnel.

Ainsi, en fonction des risques potentiels (cartes de danger) et des contraintes matérielles, les interventions sur les cours d'eau sont aujourd'hui régies par :

- des mesures préventives, telles que le maintien de la capacité d'écoulement et de l'efficacité des ouvrages de protection existants (entretien de la ripisylve, entretien des berges, gestion des matériaux charriés, reboisement des rives, faucardage du fond des lits, vidange des dépotoirs, etc.) ;

- des mesures d'aménagement du territoire (délimitation d'espaces libres inondables en cas d'événements extrêmes, de l'espace de divagation du cours d'eau, de zones de dangers, etc., au travers de plans directeurs et d'affectation);

- des mesures structurelles combinées et limitées, car, techniquement, elles se sont avérées parfois peu efficaces et parce que la protection absolue n'existe pas ;

- des mesures de réhabilitation de la dynamique fluviale et alluviale au travers d'actions sur le débit et sur le charriage (débits de restitutions, relâches de crues, interdiction de prélèvement de granulats) et sur l'espace disponible (rétablissement d'un lit de divagation).

Notons qu'une forte volonté politique s'exprime par différentes lois à incidences spatiales et environnementales, mises en place depuis les années 1990 pour une protection, une conservation et une restauration des milieux humides et plus particulièrement des plaines et des zones alluviales.

Il reste qu'aujourd'hui, la restauration ou le retour à l'état de " pré-dégradation » (Shields et al., 1999) est illusoire au vu des transformations radicales des bassins versants alpins suite au développement socioéconomique 
de ces derniers; un seuil d'irréversibilité a souvent été atteint. D'un point de vue technique, deux cas de figure sont possibles. Premièrement, si le processus $d$ 'incision dans une zone alluviale est irréversible, on procédera à une revalorisation, par création de milieux de substitution, selon les définitions de Gallandat et al. (1993) : curage et approfondissement de bras morts, création d'îles, de mares et d'étangs, détournement d'affluents. Pour être efficaces, ces mesures doivent être complétées par un entretien permanent après leur réalisation.

Secondement, dans la mesure où les processus en vigueur ne sont pas irréversibles, il importe de stabiliser et stopper l'incision des lits de cours d'eau et de réhabiliter le milieu naturel. On parle alors de revitalisation ou de restauration (ibidem $)^{4}$. Il s'agit ici d'intervenir sur la dynamique alluviale et de procéder à des actions combinées sur le débit solide et liquide, sur l'espace disponible et sur la morphologie de la zone alluviale. Les actions à mener pour rétablir un régime hydrologique satisfaisant en termes de processus de charriage, de sédimentation et d'érosion dans la zone alluviale concernent :

- l'augmentation des débits de restitution à l'aval des aménagements hydroélectriques, tout en procédant à des relâches lors de crues importantes ;

- l'augmentation du charriage, en diminuant la rétention du matériel sédimentaire dans les barrages de retenue en amont et en interdisant ou en réglementant le prélèvement de graviers dans les lits de cours d'eau;

- le démantèlement de certaines digues dans les zones alluviales afin de réactiver l'érosion latérale des berges, sources importantes de matériel sédimentaire mobilisable par la rivière.

L'élargissement de l'espace de divagation du cours d'eau et de son lit d'inondation nécessite de déplacer ou de supprimer certains ouvrages de protection contre les crues. Il faut également, parfois, abaisser les berges et le lit majeur. En général, une fois ces actions menées, une nonintervention est prônée dans la zone revitalisée.

La mise en pratique de ces actions, malgré un cadre législatif étendu, reste difficile. Agir sur les débits de restitution n'est possible légalement que pour les nouvelles concessions d'exploitation de la ressource eau, ou bien si des espaces sont protégés au niveau fédéral. Les débits solides ne sont pas concernés. Élargir l'espace d'écoulement des cours d'eau nécessite des accords avec les

\footnotetext{
${ }^{4}$ Le terme de revitalisation a été préféré à celui de restauration (cf. par exemple SER and Policy Working Group, 2002), car il correspond mieux, au moins en français, au sens de l'article 4 de l'Ordonnance fédérale 451.31 sur les zones alluviales du 28 octobre 1992, qui prévoit le rétablissement de la dynamique naturelle du régime des eaux et du charriage en vue de favoriser la diversité des espèces alluviales (cf. http://www.admin.ch/ch/f/rs/c451_31.html).
}

propriétaires riverains; le plus souvent, l'achat de terrain par l'État reste la voie la plus efficace.

\section{Les contraintes culturelles}

La mise en pratique de ces exigences impose des conditions techniques et opérationnelles spécifiques, mais aussi le dépassement d'un certain nombre de contraintes culturelles construites sur des représentations sociales diverses et parfois contradictoires des risques, de la vulnérabilité, du développement économique et des enjeux environnementaux qui lui sont associés, de l'articulation entre les pratiques locales et les enjeux globaux en matière de protection de l'environnement. Trois autres éléments interviennent dans la complexité des représentations sociales et entravent l'action sur les plaines alluviales : la mémoire des événements catastrophiques, qui agit comme un retardateur de l'action; les échelles spatiales et temporelles de l'action, car le passage de l'agir " ici et maintenant » à un agir anticipatif suppose d'arriver à faire dialoguer des mondes de tailles différentes (action individuelle versus risque global) et inscrits dans des temporalités différentes (le long terme versus le temps de l'expérience humaine) ; et les limitations d'usage des zones revitalisées, perçues souvent comme un inconvénient majeur pour les acteurs concernés. Par ailleurs, l'acceptation sociale de ces conditions se heurte à la difficulté d'obtenir un consensus auprès d'acteurs multiples, provenant d'horizons variés et défendant des intérêts souvent contradictoires.

\section{Confrontation, agencement d'intérêts contrastés et logiques plurielles d'action}

Représentants des collectivités locales, entreprises privées (usines électriques, exploitants de gravier, bureaux-conseil, etc.), administrations (offices cantonaux), experts, chercheurs, associations de professionnels et de protection de la nature, pêcheurs, forestiers, agriculteurs, touristes, éducateurs et habitants contribuent à donner forme et contenu aux principes du nouveau paradigme de gestion des zones alluviales. Promoteurs ou opposants, régulateurs ou médiateurs de ce modèle de gestion, ces acteurs se positionnent de façon inégale dans le jeu des pouvoirs, leur expertise étant de nature souvent complémentaire.

Les différentes formes d'utilisation des zones alluviales - exploitation, entretien, protection, loisirs - mobilisent des fonctions distinctes de ces espaces (produit de consommation, écosystème, zone tampon, cadre de vie, etc.) et répondent à des besoins contrastés des populations. Ainsi, des facteurs à la fois structuraux et culturels se combinent pour donner lieu à des logiques d'action multiples; ces dernières orientent par la suite les 
pratiques et les modes d'action des acteurs concernés. L'enquête sociologique (Encadré) a permis de repérer et de décrire des logiques plurielles, multidimensionnelles, d'exploitation et d'action sur ces zones, en fonction de différents éléments socioculturels, économiques et institutionnels. Elle dégage par la suite quelques composantsclés de ces logiques, tout en précisant que leur mise à plat a comme seul but de faciliter l'identification des facteurs déterminant l'action.

\section{La logique de survie}

Le modèle de gestion articulé autour de la sécurité s'est bâti au XIX ${ }^{\mathrm{e}}$ siècle et consolidé au XXe autour d'une logique de survie à plusieurs dimensions. Se légitimant par l'impératif indubitable de la protection des biens et des vies humaines contre le danger d'inondation, cette logique a concerné tout autant les besoins de garantir une sécurité alimentaire et sanitaire. $\mathrm{Si}$, avec le développement économique et technologique des sociétés occidentales, ce raisonnement sécuritaire - fondateur de toute une idéologie de protection - a perdu de son poids, il continue toutefois d'être reproduit et défendu par ses principaux adeptes : agriculteurs, ingénieurs, gestionnaires, riverains. La sécurité représentant un projet fédérateur fort, cette logique fut le moteur de l'action pendant plus de 150 ans. Avec la problématique environnementale, nous la retrouvons sous une forme renouvelée. La préoccupation pour un autre type de survie émerge : celle de l'espèce humaine, et implicitement celle des écosystèmes, projetée dans un temps long.

\section{La logique marchande}

L'intervention sur la dynamique et la morphologie des zones alluviales est souvent légitimée par son utilité économique. Ce raisonnement suppose l'attribution d'une valeur marchande aux zones alluviales et à ses produits dérivés. Cette logique sous-tend, à des échelles et avec des impacts différents sur la dynamique et la configuration des zones alluviales, des activités telles que la production hydroélectrique, l'extraction de gravier, l'exploitation agricole de parcelles (autrefois inondables), l'exploitation forestière, le ramassage du bois, la pêche, etc. Par ailleurs, des projets d'aménagement avec des incidences importantes sur le fonctionnement de ces zones (par exemple, des projets de réalisation d'un terrain de golf, d'un port, des sports nautiques et des activités de loisirs avec retombées financières, etc.) obtiennent le soutien des collectivités locales, car ils concourent à l'amélioration de l'image et de l'attrait touristique des communes.

\section{Le bien-être}

La pratique individuelle de la nature, plus particulièrement celle des zones alluviales, peut dériver d'une logique de bien-être. Elle s'accomplit dans des activités de détente (randonnées, dessin, méditation, etc.) et mobilise des qualités non utilitaristes de ces milieux, mais à forte valeur pour une société dans laquelle l'individualisation est un processus qui atteint tous les aspects de la vie sociale.

\section{La logique civique ou volontariste}

Le même processus d'individualisation de la société engage, de plus en plus, une prise de conscience et de responsabilités quant à la protection de la nature. Cette responsabilisation peut conduire non seulement à des pratiques respectueuses, mais aussi à des prises de position citoyennes en faveur de la sauvegarde des zones alluviales et à des campagnes plus larges de sensibilisation du public et d'éducation à l'environnement. On assiste ainsi à l'apparition d'une démarche civique, traduite par une participation engagée des individus.

\section{La contrainte réglementaire}

À partir des années 1990 et avec l'introduction des principes du développement durable dans la législation suisse, les exigences légales dans le domaine de la protection contre les crues imposent la prise en compte des aspects environnementaux dans les travaux d'entretien ou d'aménagement des cours d'eau. Les premiers à intégrer ces normes ont été les services cantonaux, qui jouent à la fois un rôle de promoteurs de ces nouveaux principes et de garants de leur application. Dans cette perspective, et malgré certaines différences notables entre les pratiques des autorités cantonales (reflet du contexte socioculturel, mais aussi de leur degré d'indépendance politique vis-àvis du pouvoir central), la transformation des modes de gestion des zones alluviales rentre dans une logique de conformité aux dispositions légales. L'action des acteurs économiques (principalement des exploitants d'énergie électrique et des exploitants de gravier, mais aussi des chasseurs, des pêcheurs, etc.) est aussi régularisée par des réglementations.

\section{La participation et la médiation - les deux clés de voûte du nouveau paradigme}

L'articulation d'intérêts et de logiques d'action contrastés nécessite un renouvellement de l'action publique, par la mise en place de nouvelles procédures axées sur la communication, la médiation et la participation. 


\section{La création de sphères de discussion publique interdisciplinaire}

Dans les trois cas étudiés, nous avons constaté que les instances administratives de l'État ont développé des stratégies axées sur l'information et la mise en place d'interfaces de communication et de procédures participatives. Le rôle de ces stratégies est d'inciter à une prise de conscience collective, face aux enjeux écologiques, dans la gestion des zones alluviales et de stimuler une réponse citoyenne à cet égard. Tout un travail de préparation et de concertation a été entamé au niveau des services cantonaux. Dans les trois cantons, des groupes interdisciplinaires de réflexion et de travail ont été créés. En règle générale, ces groupes réunissent des représentants des services cantonaux de protection des cours d'eau et de la nature, de la chasse et de la pêche, des forêts et de l'aménagement du territoire, mais aussi des professionnels indépendants et des bureaux d'experts privés. C'est un exercice de dialogue entre des spécialistes qui font l'effort de sortir des logiques sectorielles et qui s'appliquent à un travail de coordination de leurs activités autour d'un objectif commun d'intérêt public. Ce processus s'ouvre et s'élargit dans un deuxième temps à la consultation et à la participation d'autres acteurs. En outre, nous assistons à l'émergence graduelle d'un espace intermédiaire, de concertation, qui articule le débat institutionnel aux formes de participation de la société civile, un espace de communication entre les différentes sphères (institutionnelle, économique et sociale) d'action. Des procédures de concertation novatrices " cherchent à rendre gouvernables des situations complexes en empruntant des voies alternatives aux modes classiques de l'expertise, $\mathrm{du}$ traitement bureaucratique et réglementaire et de la délégation politique »(Salles, 2004, p. 4). Par la mise en débat public, l'organisation de conférences citoyennes ou la création de comités consultatifs, on essaye certes de rendre acceptables des solutions imposées " par le haut", mais surtout de provoquer une prise de conscience et de stimuler l'initiative des populations concernées.

Nous constatons cependant que, dans la réalité, la délégation des pouvoirs et des responsabilités vers la société civile peut, dans un premier temps, inhiber l'action et doit s'opérer en plusieurs étapes. Dans le cas de l'initiation et de la mise en œuvre des projets de revitalisation, notre étude signale plusieurs cas de figure :

- les bureaux cantonaux (offices, services) sont le plus souvent à l'origine des projets tout en assumant leur coordination;

- les bureaux privés proposent des solutions novatrices aux communes, le canton aide au financement des projets ;

- les collectivités locales cherchent des solutions à un problème existant (de protection, d'entretien ou d'aménagement) et sollicitent les autorités et les acteurs privés.

Dans chaque situation, la réalisation de projets est accompagnée d'un intense travail d'information et de médiation qui œuvre pour augmenter la connaissance objective de ces types d'aménagement et les rendre ainsi acceptables auprès du public de non-spécialistes.

\section{Le rôle des structures intermédiaires de médiation}

La médiation reste le concept-clé du nouveau modèle de gestion, intégré et codisciplinaire. Elle concourt à la conjugaison des intérêts et des efficacités (sécuritaire, économique, sociale et écologique) dans la gestion des zones alluviales. L'émergence des structures intermédiaires qui opèrent une médiation entre l'État et les dynamiques sociales témoigne de la mise en œuvre d'un « institutionnalisme centré sur les acteurs» (Mayntz et Scharpf, 1995). Ce processus s'accompagne d'un transfert de pouvoirs, de responsabilités et de compétences des « structures régulatrices » vers les « structures opératoires » (Papadopoulos, 1995 ; Lange et Braun, 2000) qui se distinguent par leur capacité à agir pour résoudre des problèmes concrets. Le rôle de médiation est assuré par les opérateurs de terrain, notamment les bureaux privés, habituellement des professionnels de l'environnement ou du risque (géographes, écologues, ingénieurs). Ils bénéficient de savoirs pluriels, sont en général initiateurs des projets et assument souvent le rôle de médiateurs sociaux dans le processus de négociation qui accompagne la procédure de réalisation des travaux de revitalisation. Ils se chargent d'agir auprès de communautés locales, d'amener les différents acteurs en présence et de médiatiser la communication entre eux. Nous avons affaire ici à une nouvelle forme de privatisation de l'expertise. Le spécialiste joue davantage un rôle d'animateur et « son aide à la décision sera investie d'un sens inédit : mettre en place les structures nécessaires à la communication entre systèmes et s'attacher à stimuler le dialogue. [...] l'expertise devient dès lors largement le domaine d'un savoir-faire pratique» (Papadopoulos, op. cit., p. 118). Un nouveau rapport au savoir, culturellement médiatisé, prend forme.

\section{Le retour d'expériences ou les itinéraires d'apprentissage collectif : l'exemple d'une revitalisation réussie}

La prise de conscience relative aux enjeux environnementaux adjacents à l'aménagement des zones alluviales se fonde indéniablement sur l'expérience. L'exemple de la revitalisation réalisée en 1999 à Grono, sur le site d'étude situé dans le canton des Grisons, permet de 
mettre en évidence les arguments qui ont rendu possible la mise en œuvre du projet ainsi que les processus d'apprentissage collectif qui s'ensuivent.

Reçu avec réticence par une partie de la bourgeoisie ${ }^{5}$ locale et des habitants riverains, ce projet est considéré aujourd'hui comme une réussite, tant par ses initiateurs que par ses opposants. Il montre comment l'interaction entre les schémas de pensée préexistants et les nouveaux modes d'action participe à la transformation des représentations sociales et des comportements des acteurs locaux.

Plusieurs éléments ont contribué à la concrétisation de cette revitalisation. L'argument sécuritaire a fonctionné au mieux pour faire avancer ce projet; en répondant à un besoin réel de réaménagement, sa réalisation a représenté aussi l'occasion de promouvoir les avantages écologiques des revitalisations et de familiariser les populations et les autorités locales à ce genre d'interventions. En outre, même si les modèles sécuritaires ont changé, ce sont les mêmes acteurs qui assument le rôle de pionniers et de promoteurs des mesures de revitalisation - en l'occurrence, les offices cantonaux pour l'aménagement des cours d'eau, chargés de la protection contre les crues. L'élargissement du lit de la rivière permet de dissiper l'énergie de celle-ci et, ainsi, de diminuer la force des crues. L'argument sécuritaire, ancré dans une logique d'autorité technocratique et d'intervention top-down, est plus facilement recevable, même quand il s'agit de proposer des techniques différentes, respectueuses de la nature. Nous remarquons aussi que la biodiversité, et donc l'argument écologique, ne pèse pas encore très lourd, constituant davantage l'objet d'une forte négociation. Ceci est dû, en partie, au fait que le rôle et l'importance des revitalisations pour le fonctionnement des écosystèmes sont encore assez mal connus. Les services administratifs de protection de la nature ont participé à la mise en place de procédures de négociation, mais ils disposent de ressources financières limitées et sont encore relativement impuissants pour imposer ces mesures. Ils ont cependant un rôle important dans l'organisation de campagnes de sensibilisation et d'information. Des groupes d'experts, des représentants de ProNatura Suisse ou du WWF contribuent en outre à apporter des arguments scientifiques quant aux bénéfices des revitalisations et agissent surtout en tant que groupes de pression.

\footnotetext{
${ }^{5}$ Le terme « bourgeoisie» renvoie ici à la collectivité de droit public qui regroupe les détenteurs du droit de cité d'une localité. En Suisse, dans certains cantons, la commune bourgeoise est distincte de la commune politique. Elle est généralement propriétaire de biens qu'elle gère elle-même ou à travers une corporation bourgeoise. Elle porte différents noms selon les régions (par exemple, patriziato au Tessin ou vischnanca burgaisa aux Grisons).
}

Les arguments économiques et financiers se sont avérés également des régulateurs forts dans la mise en œuvre de projets. Dans le cas de Grono, le fait de proposer des coûts moins élevés par rapport aux travaux traditionnels de protection contre les crues a contribué à persuader les élus locaux de passer à l'acte. Par ailleurs, le financement des travaux de revitalisation est en général assuré dans une grande proportion par le canton ou la Confédération (faisant partie d'une logique incitative); d'autres sources de financement peuvent toutefois être attirées, des entreprises privées et des collectivités locales s'y associant si elles y trouvent leur intérêt. Ce fait est crucial pour garantir la pérennité de la démarche. À Grono, une monnaie d'échange intéressante a été représentée par la possibilité d'extraire du gravier suite aux manœuvres nécessaires à ces travaux de revitalisation. De plus, nous constatons une articulation prometteuse entre les buts économiques et écologiques de cette activité : d'une part, le gravier extrait pour la réalisation de la revitalisation de Grono a été vendu, en partie importante, au profit des Chemins ferroviaires fédéraux pour faire du béton pour les transversales alpines; $\mathrm{d}$ 'autre part, l'exploitation respectueuse et raisonnée des dépôts de gravier dans les zones revitalisées est devenue un outil d'entretien des cours d'eau dans un souci d'accroître la sécurité.

Sur le plan symbolique, le réaménagement de ces espaces auparavant « résiduels » (ici la zone alluviale était devenue une décharge d'ordures) a conduit au rétablissement du lien coupé avec la rivière. La création d'espaces de transition et de loisirs, accessibles au grand public, contribue aujourd'hui à un réinvestissement social de ces lieux. Le fait de se réapproprier l'espace alluvial, d'intégrer et de partager cette expérience au quotidien, concourt au fur et à mesure à un changement des pratiques et des mentalités.

\section{Pour conclure}

Cette recherche interdisciplinaire a permis de mettre au jour la complexité du système de contraintes - hydrologiques, écologiques et culturelles - qui délimite l'action publique, ainsi que les processus participatifs à travers lesquels se négocient l'acceptation et la mise en œuvre du nouveau paradigme de gestion des zones alluviales. Nous avons pu constater l'alignement de la gestion de ces zones sur un courant dominant dans l'approche environnementale, caractérisée par la centralité de la participation, de la négociation et du consensus dans des procédures démocratiques, participatives, flexibles et transparentes. Nous pensons cependant que rester à ce niveau d'analyse serait un piège pour la recherche, car, malgré la mise en place de « bonnes procédures ", encore peu de travaux de revitalisation ont été réalisés en Suisse. De surcroît, les spécialistes constatent 
qu'en dépit de la prise de conscience qui accompagne le changement de modèle de gestion, les espaces naturels continuent d'être fortement morcelés, la disparition des espèces n'est pas stoppée, et les causes de cette dégradation des milieux ne disparaîtront pas, au contraire. La pression démographique et la permanence des priorités de l'économie sur l'écologie sont de plus en plus contraignantes, malgré des stratégies qui à long terme devraient améliorer cette situation.

Dès lors, nous pensons que les scientifiques doivent s'intéresser à la fois aux conditions socioculturelles d'acceptation et d'application du nouveau paradigme, et aux changements que les nouveaux modes d'action et les procédures sous-jacentes produisent dans les faits. Il faudrait, entre autres, s'interroger sur les conditions d'une inscription durable des pratiques sécuritaires respectueuses de la nature dans l'agir collectif, capable de produire à terme un changement profond des mentalités.

\section{Références}

Amoros, C., Petts, G.E., 1993. Hydrosystèmes fluviaux, Paris, Masson.

Bennett, R.J., Chorley, R.J., 1978. Environmental Systems: Philosophy, Analysis and Control, London, Methuen and Co. Ltd.

Bravard, J.-P., Amoros, C., Pautou, G., 1986. Impact of civil engineering works on the successions of communities in a fluvial system, Oikos, 47, 92-111.

Chorley, R.J., Kennedy, B.A., 1971. Physical Geography: A Systems Approach, London, Prentice-Hall.

Décamps, H., Décamps, O., 2002. Ripisylves méditerranéennes, Arles (Le Sambuc), Tour du Valat.

Descroix, L., Gautier, E., 2002. Water erosion in the southern French Alps: climatic and human mechanisms, Catena, 50, 1, 53-85.

Ellenberg, H., 1988. Vegetation Ecology of Central Europe, Cambridge, Cambridge University Press.

Gallandat, J.-D., Gobat, J.-M., Roulier, C., 1993. Cartographie des zones alluviales d'importance nationale, Cahier de l'environnement, 199.

Girel, J., 1991. Aménagements anciens et récents. Incidences sur l'écologie d'un corridor fluvial : la Leysse dans le bassin chambérien, Revue d'écologie alpine, 1, 81-95.

Hary, N., Nachtnebel, H.-P. 1989. Ökosystemstudie Donaustau Altenwörth: Veränderungen durch das Donaukraftwerk Altenwörth, Innsbruck, Universitätsverlag Wagner.

Landon, N., Piégay, H., Bravard, J.-P., 1998. The Drôme river incision (France): from assessment to management, Landscape and Urban Planning, 43, 1-3,119-131.

Lange, S., Braun, D., 2000. Politische Steuerung zwischen System und Akteur: Eine Einführung, Opladen, Leske und Budrich.

Liébault, F., Piégay, H., 2001. Assessment of channel changes due to long-term bedload supply decrease, Roubion River, France, Geomorphology, 36, 3-4, 167-186.

Malavoi, J.-R., 1998. Détermination de l'espace de liberté des cours d'eau, Lyon, Agence de l'eau Rhône Méditerranée Corse.

Mayntz, R., Scharpf, F., 1995. Steuerung und Selbstorganisation in staatsnahen Sektoren, in Mayntz R., Scharpf F. (Eds.),
Gesselscaftiliche Selbstregelung und politische Steuerung, Frankfurt am Main, Campus, pp. 16-17.

Müller-Wenk, R., Huber, F., Kuhn, N., Peter, A., 2004a. Landnutzung in potenziellen FliessgewässerAuen : Artengefährdung und Ökobilanzen, Bern, Bundesamt für Umwelt, Wald und Landschaft (http://www.bafu.admin.ch/php/modules/shop/files/pdf/ phpW74XAL.pdf).

Müller-Wenk, R., Huber, F., Kuhn, N., Peter, A., 2004b. Riverine Floodplain Use and Environmental Damage: Final Report, Bern, Swiss Agency for the Environment, Forests and Landscape (http://www.bafu.admin.ch/php/modules/shop/files/pdf/ phpYkxTHL.pdf).

Naiman, R.J., Bilby, R.E., 1998. River Ecology and Management: Lessons form the Pacific Coastal Ecoregion, New York, Springer.

Naiman, R.J., Décamps, H., McClain, M., 2005. Riparia: Streamside Communities, San Diego, Elsevier Academic Press.

Nedelcu, M., Hainard, F., 2006. Pour une écologie citoyenne: risques environnementaux, médiations et politiques publiques, Paris, L'Harmattan.

Office fédéral de la statistique [OFS], 2002. Environnement suisse : statistiques et analyses. Rapport OFS, Neuchâtel (http://www.bfs.admin.ch/bfs/portal/fr/index/themen/ raum_und_umwelt/uebersicht/blank/analysen_berichte/ umwelt2002/01.htm).

Papadopolous, Y., 1995. Complexité sociale et politiques publiques, Paris, Montschrestien.

Pautou G., 1988. Perturbations anthropiques et changements de végétation dans les systèmes fluviaux. L'organisation du paysage fluvial rhodanien entre Genève et Lyon, Documents de cartographie écologique, XXXI, 73-96.

Pautou, G., Girel, J., Borel, J.-L., 1992. Initial repercussions and hydroelectric developments in the French Upper Rhône Valley: a lesson for predictive scenarios propositions, Environmental Management, 16, 2, 231-242.

Pautou, G., Girel, J., Peiry, J.-L., Hugues, F., Richards, K., Foussadier, R., Garguet-Duport, B., Harris, T., Barsoum, N., 1996. Les changements de végétation dans les systèmes fluviaux. L'exemple du Haut-Rhône et de l'Isère dans le Grésivaudan, Revue d'écologie alpine, 3, 41-66.

Peiry, J.-L., Salvador, P.-G., Nouguier, F., 1994. L'incision des rivières dans les Alpes du Nord : état de la question, Revue de géographie de Lyon, 69, 1, 47-56.

Piégay, H., 1997. Principes nouveaux en matière de gestion des marges de rivières en tresses (exemple de la moyenne vallée du Giffre, Haute-Savoie, France), Revue de géographie alpine, 2, 2, 33-48.

Piégay, H., Pautou, G., Ruffinoni, C., 2003. Les Forêts riveraines des cours d'eau : écologie, fonctions et gestion, Paris, Institut pour le développement forestier.

Salles, D., 2004. Les procédures collaboratives : démocratie et performance environnementale, in Gendron, C., Salles, D., Vaillancourt, J.-G. (Eds), Environnement et développement durable : pratiques individuelles et collectives, actes du colloque organisé dans le cadre du XVII ${ }^{e}$ Congrès de l'AISLF, Tours, 5-9 juillet 2004, 3-12.

Schmid, F.S., 2002. Grande est la détresse, plus grand encore est l'amour du prochain. L'entraide des inondations de 1868 dans l'espace alpin, in Pfister, C. (Ed.), Le Jour d'après : surmonter les catastrophes naturelles, le cas de la Suisse entre 1500 et 2000, Bern, Haupt, 87-101. 
Schumm, S.A., 1977. The Fluvial System, New York, John Wiley $\&$ Sons.

SER and Policy Working Group, 2002. The SER Primer on Ecological Restoration (http://www.ser.org/content/ ecological_restoration_primer.asp).

Shields, F. D., Brookes, A., Haltiner, J., 1999. Geomorphological approaches to incised stream channel restoration in the United States and Europe, in Darby, S.E., Simon, A. (Eds), Incised River Channels, New York, John Wiley \& Sons, 371394.

Surian, N., Rinaldi, M., 2003. Morphological response to river engineering and management in alluvial channels in Italy, Geomorphology, 50, 4, 307-326.

Tockner, K., Ward, J.V., 1999. Biodiversity along riparian corridors, Archiv für Hydrobiologie - Supplementbände, 115, 3, 293-310.

Reçu le 28 avril 2006. Accepté le 7 décembre 2006.
Vannote, R.L., Minshall, G.W., Cummins, K.W., Sedell, J.R., Cushing, C.E., 1980. The river continuum concept, Canadian Journal of Fisheries and Aquatic Sciences, 37, 1, 130-137.

Vischer, D., 2003. Histoire de la protection contre les crues en Suisse : des origines jusqu'au $19^{\mathrm{e}}$ siècle, Berne, OFEG.

Ward, J.V., 1989. The four dimensional nature of lotic ecosystems, Journal of the North American Benthological Society, 8, 1, 2-8.

Ward, J.V., Burgherr, P., Gessner, M.O., Malard, F., Robinson, C.T., Tockner, K., Uehlinger, U., Zah, R., 1998. The Val Roseg Project: habitat heterogeneity and connectivity gradients in a glacial floodplain system, in Kovar, K., Tappeiner, U., Peters, N.E., Craig, R.G. (Eds), Hydrology, Water Resources and Ecology in Headwaters, Wallingford, IAHS Press, 425-432.

Willi, H.P., Jordan, J.-P., Roth, U., Frei, B., 2001. Protection contre les crues des cours d'eau, Berne, OFEG.

To access this journal online: www.edpsciences.org 